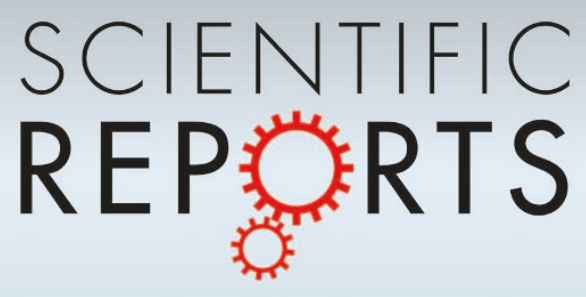

OPEN

SUBJECT AREAS:

ELECTRICAL AND

ELECTRONIC

ENGINEERING

ELECTRONIC PROPERTIES AND MATERIALS

Received

16 July 2013

Accepted

11 November 2013

Published

6 December 2013

Correspondence and requests for materials should be addressed to S.P.L. (shunpu.li@york. ac.uk) or S.R. (saibal.

roy@tyndall.ie)

\section{Electrical/optical dual-function redox potential transistor}

\author{
Shunpu Li ${ }^{1,2}$, Wensi Wang ${ }^{1}$, Ju Xu ${ }^{1}$, Daping Chu ${ }^{3}$, Z. John Shen ${ }^{4} \&$ Saibal Roy ${ }^{1,5}$
}

\begin{abstract}
${ }^{1}$ Microsystems Center, Tyndall National Institute, Dyke Parade, Cork, Ireland, ${ }^{2}$ Department of Electronics, University of York, Heslington, York YO10 5DD, UK, ${ }^{3}$ Electrical Engineering Division, University of Cambridge, Cambridge CB3 OFA, UK, ${ }^{4}$ Department of Electrical and Computer Engineering, Illinois Institute of Technology, Chicago, IL 60616, USA, ${ }^{5}$ Presently at Department of Electrical Engineering, Stanford University, Stanford, CA 94305-9505, USA.
\end{abstract}

We demonstrate a new type of transistors, the electrical/optical "dual-function redox-potential transistors", which is solution processable and environmentally stable. This device consists of vertically staked electrodes that act as gate, emitter and collector. It can perform as a normal transistor, whilst one electrode which is sensitised by dye enables to generate photocurrent when illuminated. Solution processable oxide-nanoparticles were used to form various functional layers, which allow an electrolyte to penetrate through and, consequently, the current between emitter and collector can be controlled by the gate potential modulated distribution of ions. The result here shows that the device performs with high ON-current under low driving voltage $(<1 \mathrm{~V})$, while the transistor performance can readily be controlled by photo-illumination. Such device with combined optical and electrical functionalities allows single device to perform the tasks that are usually done by a circuit/system with multiple optical and electrical components, and it is promising for various applications.

T ransistor, one of the most important inventions in the $20^{\text {th }}$ century, has always been a research focus in both the academia and industry. Research concerning transistors has been very active in several domains. Significant efforts have been provided exploring new functional materials like inorganic crystalline semiconductors $^{1-5}$, small molecule and polymer semiconductors ${ }^{6-11}$, nanotubes ${ }^{12,13}$ and recently graphenes ${ }^{14,15}$. The revolutionary achievements of transistors can also be attributed to the adoption of novel device architectures and operating principles, such as the application of field effect $^{16,17}$, dynamically doping/dedoping ${ }^{18}$ etc. Low-cost fabrication based on solution process has also drawn considerable attention in the past decade ${ }^{6,11,19,20}$. Solution processable large-scaled organic transistor based circuit, with a great potential to reduce fabrication cost, has already been demonstrated ${ }^{21}$.

An important forefront of transistor research resides in the area of multi-functionality devices, which allow a single device to replace a circuit block of components in different domains such as electronics and optics. A reduction in the number of components will lead to increased system package density with increased functionality and consequently improved system performance. For example, a light emitting transistor which combines the functionalities of both a transistor and a light emitting diode (LED) is promising due to reduction of system complexity and cost in optical communication modules and electrically driven lasers ${ }^{22,23}$. Despite the significant progress made so far in this field, exploration of new types of transistors, novel materials and low cost manufacturing technologies is still continuing. Here we use solution processable oxide nanoparticles to realise a low cost and environmentally stable transistor in which the electrical current between two electrodes can be controlled by a third electrode through tuning redox process. Besides operating as a normal transistor (electrically controlled) the device can also respond to illumination as one of its electrodes is sensitised by dyes which converts photons into charge carriers.

\section{Results}

Figure 1 shows a schematic illustration of the proposed device structure (Fig. 1a), fabrication process (Fig. 1b) and transistor with common emitter configuration (Fig. 1c). The gate electrode is made of fluorine doped tin oxide (FTO) layer coated with a $30 \sim 50 \mathrm{~nm}$ thick bulk $\mathrm{TiO}_{2}$ film. To realize photo-response a layer of n-type semiconductor nanoparticle $\left(\mathrm{TiO}_{2}\right)$ is deposited onto the FTO and sensitized with dyes. The porous emitter electrode is formed from indium tin oxide (ITO) nanoparticles in between the gate and collector electrodes (detailed in method section). A $\mathrm{ZnO}$ nanoparticle layer is used as a supporting layer to isolate the emitter from the Pt-catalyst coated FTO collector. Electrolyte is filled into the space between the gate and collector. Figures $2 \mathrm{a}$ and 


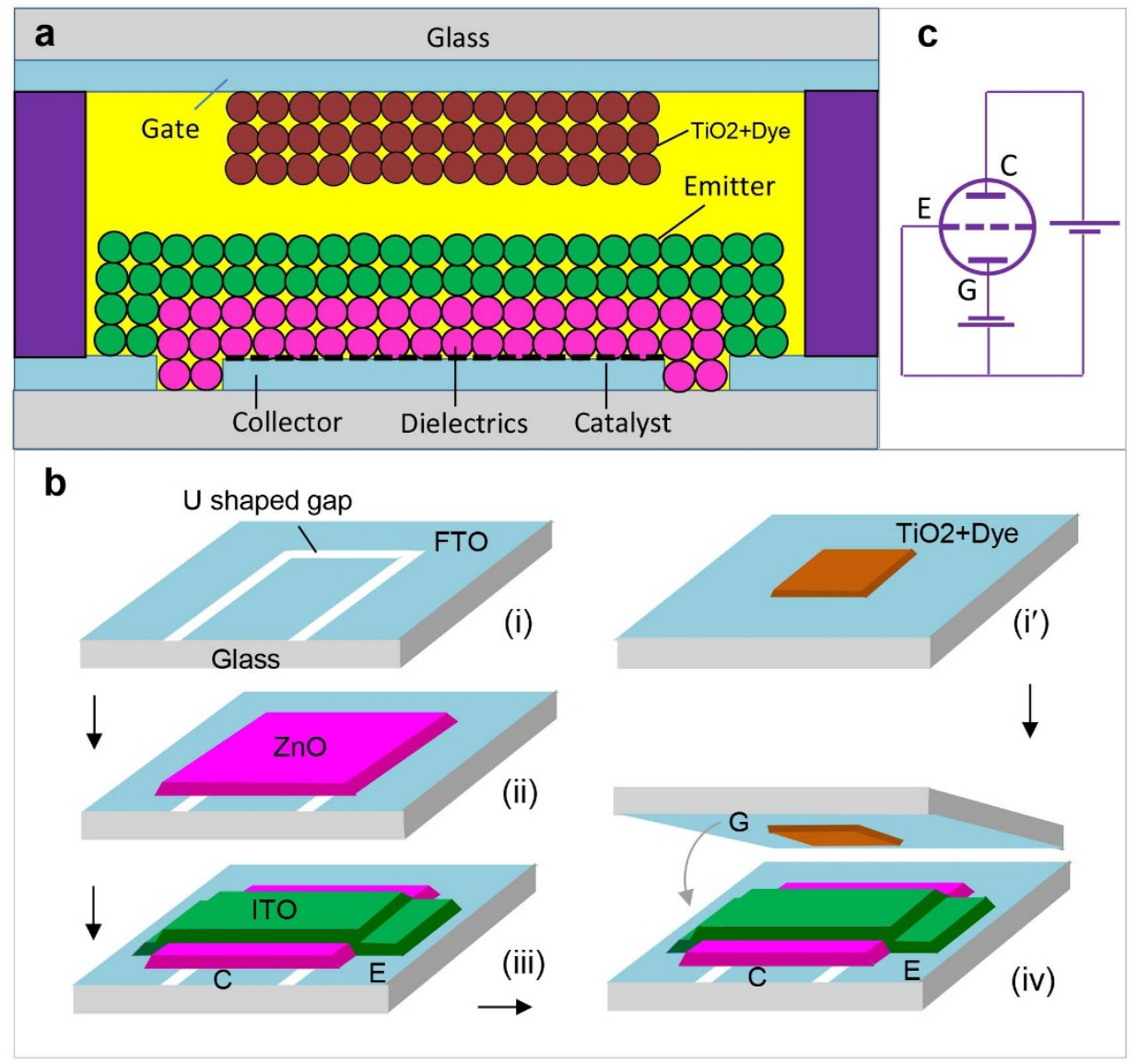

Figure 1 Schematic illustrations of (a) the proposed device architecture, (b) fabrication process, and (c) the device with common emitter configuration.

$2 \mathrm{~b}$ show typical electrodes fabricated and Fig. 2c shows a scanning electron microscope (SEM) image of the $\mathrm{ZnO}$ and ITO films coated on FTO substrate where the colors generated by energy-dispersive Xray (EDX) analysis represent the corresponding materials (Green: In, Pink: Zn, and Blue: Sn).

The devices were characterized using Keithley 2400 multimeters, an oscilloscope, and a xenon light source with adjustable light intensities.
Figure 3 shows output curves of collector current-voltage $\left(\mathrm{I}_{\mathrm{C}}-\mathrm{V}_{\mathrm{C}}\right)$ taken at various gate voltages $\left(\mathrm{V}_{\mathrm{G}}\right)$ and transfer curves of $\mathrm{I}_{\mathrm{C}}-\mathrm{V}_{\mathrm{G}}$ under constant collector voltage (inset, $\mathrm{V}_{\mathrm{C}}=0.8 \mathrm{~V}$ ). The measurement was done using a common emitter configuration (Fig. 1c) under dark (Fig. 3a) and illumination with 100Klux light (Fig. 3b). The obtained characteristics of our devices are rather similar to that of the well-developed triode (see supporting information, Fig. 1).
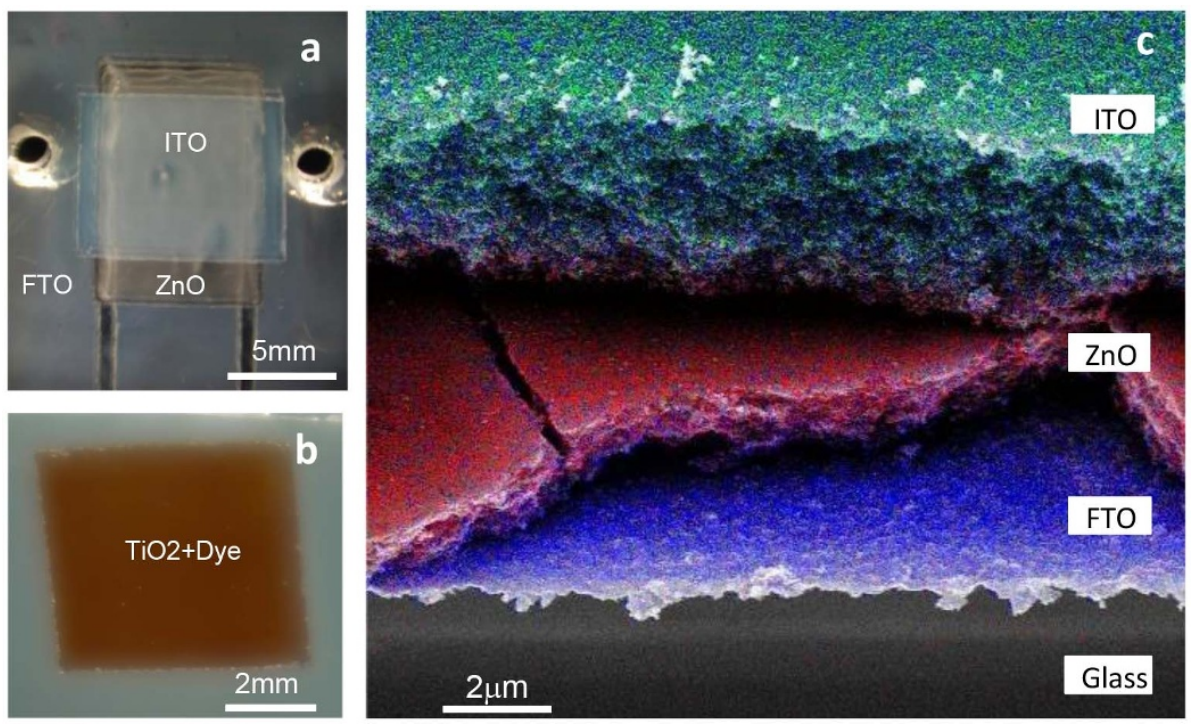

Figure $2 \mid$ Optical images of (a) the emitter/collector electrodes and (b) the gate/photo-electrode and (c) SEM image of the ZnO and ITO films coated on a FTO/glass substrate. The colors generated by EDX analysis represent the corresponding materials as: Green: In, Pink: Zn, Blue: Sn. 


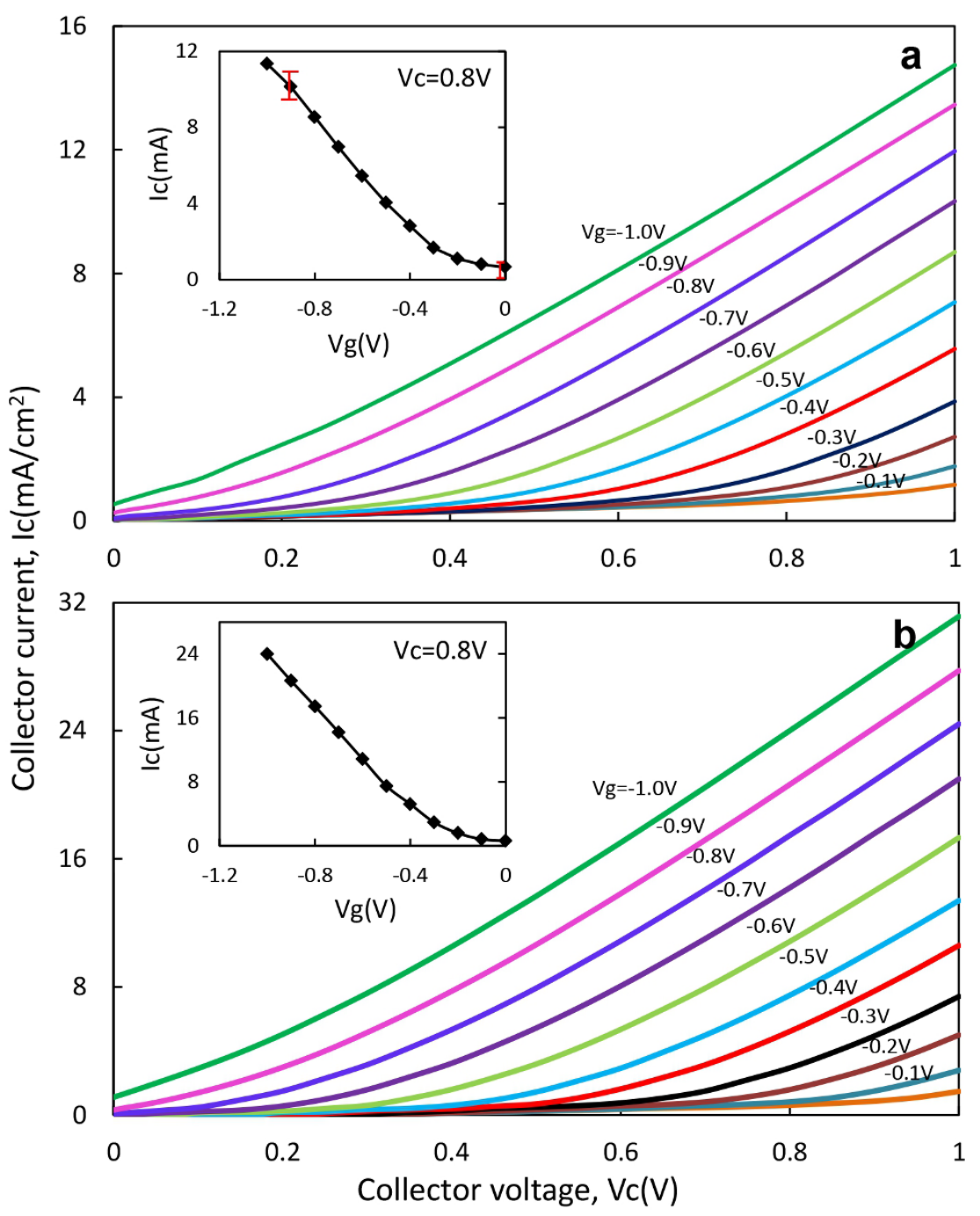

Figure $3 \mid$ Output characteristic defined by collector current-voltage $\left(\mathrm{I}_{\mathrm{C}}-\mathrm{V}_{\mathrm{C}}\right)$ curves taken under various gate voltage $\mathrm{V}_{\mathrm{G}}$ and a transfer characteristics defined by $\mathrm{I}_{\mathrm{C}}-\mathrm{V}_{\mathrm{G}}$ curve under $0.8 \mathrm{~V}$ collector voltage (inset). The device was measured with common emitter configuration (shown in Fig. 1b) under dark (a) and illumination with 100Klux of xenon light (b).

However the device we demonstrated here does not require a hot cathode, the drive voltage is two orders of magnitude lower, and can be easily miniaturized to micro-scale. The measured dark current of gate is less than $\sim 0.001 \mathrm{~mA} / \mathrm{cm} 2$ which is the leakage current caused by the reduction of ions on the exposed FTO surface due to the imperfection of the thin bulk $\mathrm{TiO}_{2}$ film. The small dark current of gate reveals that the device functions as a "field effect" or "redox potential effect" transistor. Currently achieved on/off current ratio of the transistor is $20 \sim 100$ which can be further increased by improving the quality of the $\mathrm{ZnO}$ particle film. Data scattering from same batch of devices ( 6 devices per batch) is indicated on transfer curve in Fig. 3a for ON and OFF current. The scattering of ON-current is $\sim 10 \%$, but the data scattering of OFF-current can be several times which causes the overall scattering of the ON/Off current ratio. The high ON-current of the devices is mainly attributed to the vertical channel architecture applied, and the drain current is much higher when the devices are illuminated by light. The architecture and principle of field control of the device are different from the standard field effect transistor, and hence the field effect mobility of the device cannot be extracted from existing formula. Mobility of ions in our devices was estimated to be $\sim 10^{-4} \mathrm{~cm}^{2} / V . S$. The estimation is done according to the procedure described by Koneshan et $\mathrm{al}^{24}$ where mobility of ions, such as $\mathrm{I}^{-}$, in solutions was given. The device current is proportional to charge mobility, thus finding a new electrolyte with higher charge mobility is beneficial to improve the performance.

Figures $4 \mathrm{a}$ and $4 \mathrm{~b}$ show dark and photo responses of a transistor measured with a common gate configuration (inset of Fig. $4 \mathrm{~b}$ ) where the collector current $\left(I_{C}\right)$ is measured as a function of the emitter voltage $\left(V_{E}\right)$ while the gate is grounded. As shown in Fig. $4 \mathrm{a}$ and $4 \mathrm{~b}$, the conductance defined by $d I_{C} / d V_{E}$ changes with the bias applied on the collector $V_{C}$. When $V_{C} \sim 0$ a near linear relation between $I_{C}$ and $V_{E}$ is observed, while when a bias $V_{C}$ is applied a diode behavior is seen. In both cases the curves are strongly modified and displaced when the device is illuminated. The displacement of curves, characterized by voltage shift $\left(\Delta V_{L}\right)$ and current shift $\left(\Delta I_{L}\right)$, increases with the increase of the light intensity.

Functions that usually cannot be performed with normal transistors are anticipated with the transistor demonstrated here. For instance, a single such device can function as photo-enabled current switch which is generally realized by a multi-component circuit (photo-conductor + potential shift circuit, see supporting information, Fig. 2). Fig. 4c illustrates the principle of performing such a function. The difference of voltage shift of $V_{E}$ lines (see Fig. 4c) under dark $T_{d}$ and illumination condition $T_{L}$ allows to identify a voltage $\left(\delta V_{E}\right)$ under which two collector currents with equal current amplitude but opposite polarity are found. Figure $4 \mathrm{~d}$ shows the current output under an applied $-50 \mathrm{mV}$ bias to the emitter while a 

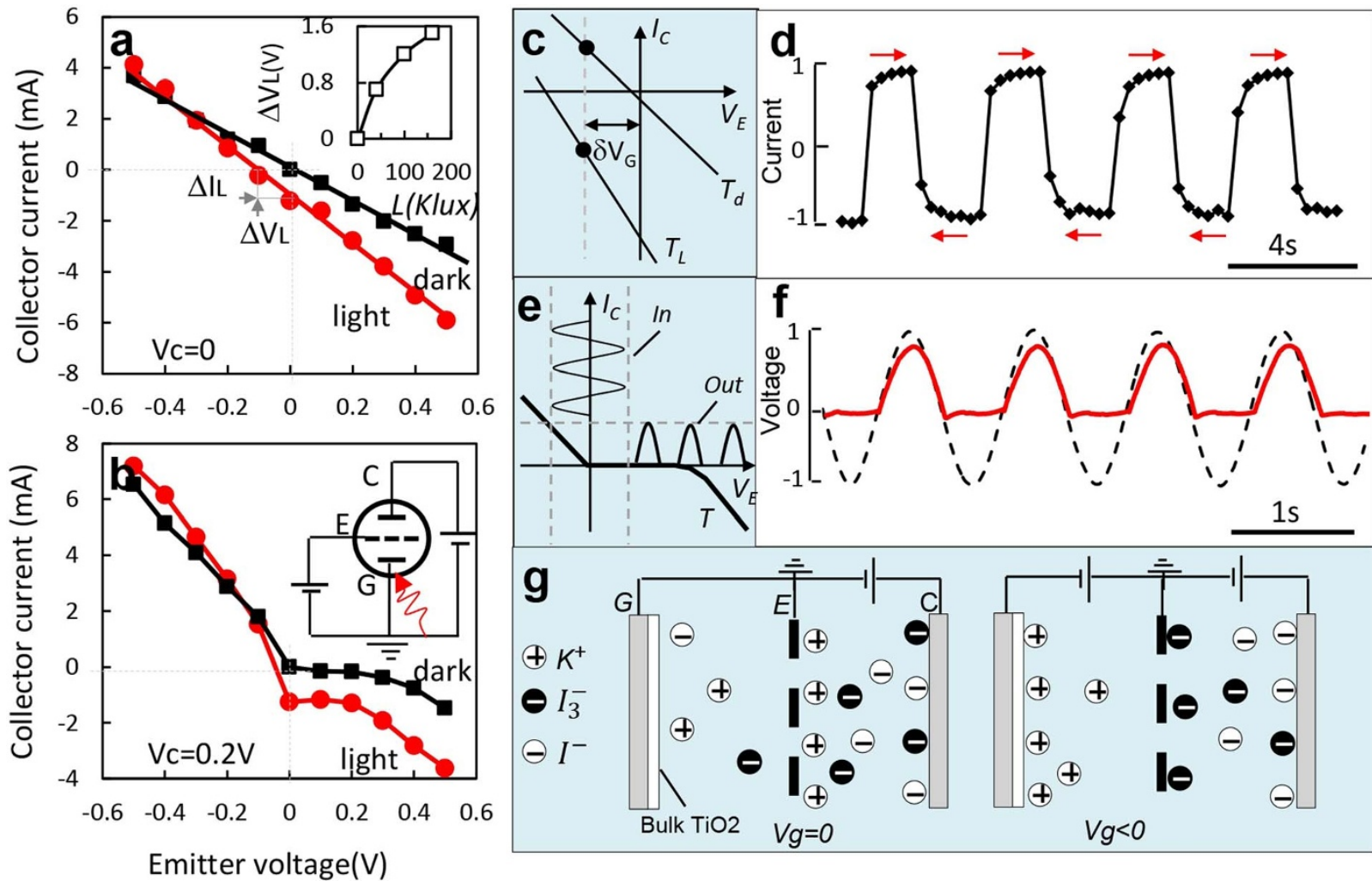

Figure $4 \mid$ (a), (b) Dark and photo-response of a transistor measured with common gate configuration (see inset of b) at $\mathrm{Vc}=0$ and $0.2 \mathrm{~V}$, respectively; (c), (d) A schematic illustration of principle of performing as photo current-switcher and experimental result of this function. The red arrows indicate different current directions when the device performs with and without illumination; (e), (f) A schematic illustration of the input and output waveforms of the device, performing as rectifier and experimental result of this function; (g) A schematic illustration of proposed mechanism of emitter-collector current change with applied gate voltage.

mechanical shutter is used to block an illuminated light periodically. The reversing of current polarity with photo-illumination demonstrates the switching function of the device. From Fig. $4 \mathrm{~b}$, it shows that a linear relation is maintained for negative emitter voltage and current cutoff happens when the emitter voltage becomes positive. This unambiguously shows that the transistor characteristic in Fig. 3 is indeed originated from a nonlinear conductance behavior which is also the basis of many other important electronic devices like diode, triode, bipolar junction transistor etc. To further clarify this, a rectification function was demonstrated. Fig. 4e shows how an input sinusoidal signal can be rectified and Fig. 4 f shows a typical half-wave rectification output obtained when a sinusoidal voltage signal is applied to the emitter with $0.2 \mathrm{~V}$ dc bias to the collector.

\section{Discussion}

The output curve with near exponential characteristics under a given gate voltage (see Fig. 3) can be qualitatively understood by the Tafel equation which describes variation of current density $i$ with applied potential $\Delta V$ on an electrochemical system ${ }^{25}$ :

$$
i=n F k \exp \left(\frac{\alpha n F \Delta V}{R T}\right),
$$

Where $n \sim$ the number of electrons involved in the electrode reaction, $F \sim$ Faraday constant, $k \sim$ rate constant for electrode reaction, $R \sim$ universal gas constant, $\alpha \sim$ charge transfer coefficient and $T \sim$ absolute temperature. For small applied voltages a linear $I-V$ relation is maintained. The gating effect (transfer characteristics of transistor) can be explained by controlling ion blockage of reduction process in the porous electrode. The reduction at the porous electrode and oxidation process at the collector electrode can be described as:

$$
\begin{aligned}
& I_{3}^{-}+2 e^{-} \rightarrow 3 I^{-} \\
& 3 I^{-}-2 e^{-} \rightarrow I_{3}^{-}
\end{aligned}
$$

The gating process in the common emitter configuration is shown in Fig. 4g. When a positive voltage is applied to the collector while the gate voltage remains zero (left of Fig. $4 \mathrm{~g}$ ), the reduction of triiodide is largely prohibited by adsorption of potassium ions on emitter material surface due to the nature of positive charge of $\mathrm{K}^{+}$. When a negative gate bias is applied (right of Fig. $4 \mathrm{~g}$ ) the $\mathrm{K}^{+}$drifted to gate from the emitter surface and the reduction of triiodide can take place through thermal diffusion. Thus, the device is switched-on by a negatively applied gate-voltage and behaves like a p-type transistor.

Previously there have been efforts to fabricate electronic devices by using electrochemical process. For instance Wang et al. have demonstrated phototransistors based on dye sensitized solar cell structure, however no electrically gating has been achieved ${ }^{26-28}$. Although transistor that responds both electrically and optically has also been demonstrated previously, it was based on standard field effect transistor configuration which is fundamentally different from the work demonstrated here ${ }^{29}$.

The emitter and collector electrodes have a low charge transfer resistance (to/from electrolyte). In contrast, the gate electrode has a higher charge transfer resistance which is realized by surface treatment with the thin bulk $\mathrm{TiO}_{2}$ film and this ensures a minimization of gate current induced by reduction reaction on it. As the bulk $\mathrm{TiO}_{2}$ film is too thin $(30 \sim 50 \mathrm{~nm})$ to hold high gate voltage, increasing gate modulation capacity requires a new device architecture where the synthetized $\mathrm{TiO}_{2}$ can be fabricated on emitter electrode while an insulator film is deposited onto the gate. The device structure with 
stacked-layers allows a high drain current density and to avoid lithographically patterning of small lateral channels of transistors which in turn allows the use of current printing technology with $\sim 10 \mu \mathrm{m}$ resolution to fabricate transistors with micron size. Potentially there is a huge space to reduce the fabrication cost further. For normal function of transistor (electrically control only) the $\mathrm{TiO}_{2}$ particle film and dye application could be eliminated and the Pt layer can be replaced by much cheaper materials, like carbon. Here the basic principle of the device is demonstrated by a well-known electrolyte which could be replaced by other materials, like solid electrolyte, which enable further miniaturization of the devices and improvement of its performance and stability. Nanoparticle materials, like $\mathrm{ZnO}$, can also be further developed or replaced to improve the $\mathrm{ON} /$ OFF current ratio $\left(>10^{4}\right)$ and this can be done by using highly resistive, smaller sized $(\sim 5 \mathrm{~nm})$ nanoparticle with better film forming property to avoid leakage induced by trapping of the subsequently coated conductive nanoparticle into the voids or defects. The lifespan of the fabricated devices should be similar to that of DSSC devices, and a typical DSSC life-time is estimated to be longer than 15 years under harsh out-door condition ${ }^{30}$. In addition, the devices demonstrated here have further proven the potentials of nanomaterial-based electrical/optical devices ${ }^{31-33}$.

\section{Methods}

A FTO film carried on glass substrate was patterned by wet etching using $2 \mathrm{M} \mathrm{HCl}$ aqueous solution with assistance of zinc powder to define a " $U$ " shaped gap to isolate the collector and contact pad of the emitter (Fig. $1 \mathrm{~b}(\mathrm{i})$ ). Then, Pt catalyst precursor (Platisol, Solaronix) was drop-casted onto the structured substrate and dried for $10 \mathrm{~min}$ at ambient condition and subsequently annealed at $450^{\circ} \mathrm{C}$ for $30 \mathrm{~min}$ to convert the precursor to Pt catalyst. Then a $1.5 \mu \mathrm{m}$ thick $\mathrm{ZnO}$ film was formed by spin-coating from its water based nanoparticle $(\sim 35 \mathrm{~nm})$ suspension onto the $\mathrm{Pt}$ coated FTO/glass substrate and baked at $150^{\circ} \mathrm{C}$ for $5 \mathrm{~min}$ (Fig. $1 \mathrm{~b}(\mathrm{ii})$ ). Next, a $4 \mu \mathrm{m}$ thick ITO film was deposited by spin-coating from isopropanol based colloidal suspension $(\sim 50 \mathrm{~nm})$ and baked at $150^{\circ} \mathrm{C}$ for $5 \mathrm{~min}$ (Fig. $1 \mathrm{~b}$ (iii)). For the preparation of the photosensitive electrode (also gate) oxygen plasma cleaned FTO/glass substrate was treated in $0.05 \mathrm{~mol} \mathrm{TiCl}_{4}$ aqueous solution for 1 hour at $80^{\circ} \mathrm{C}$ to form $30 \sim$ $50 \mathrm{~nm}$ thick amorphous $\mathrm{TiO}_{2}$ film and the film was crystallized at $450^{\circ} \mathrm{C}$ for $30 \mathrm{~min}$. $\mathrm{TiO}_{2}$ nanoparticle $(15 \sim 20 \mathrm{~nm})$ paste/suspension is deposited on the FTO substrate by screen-printing and baked at $120^{\circ} \mathrm{C}$ for $10 \mathrm{~min}$ to produce a $6 \mu \mathrm{m}$ thick $\mathrm{TiO}_{2}$ nano-particle film (Fig. $1 b\left(i^{\prime}\right)$ ). The lateral dimensions of oxide films used here were defined by adhesion type masks with windows of dimensions of $8 \mathrm{~mm} \times 10 \mathrm{~mm}$ ( $\mathrm{ZnO}), 10 \mathrm{~mm} \times 8 \mathrm{~mm}$ (ITO), and $6 \mathrm{~mm} \times 6 \mathrm{~mm}\left(\mathrm{TiO}_{2}\right)$, respectively. After calcinations of these oxide films at $450^{\circ} \mathrm{C}$ for $30 \mathrm{~min}$, the $\mathrm{TiO}_{2}$ particle film was submerged into cis-diisothiocyanato-bis $\left(2,2^{\prime}\right.$-bipyridyl-4,4' -dicarboxylato) ruthenium(II) bis(tetrabutylammonium) (N719) dye solution [0.3 mM in acetonitrile + tert-butanol $(1: 1)$ ] for 12 hours and then rinsed using acetonitrile. After bonding the gate and emitter/collector electrodes with a $60 \mu \mathrm{m}$ thick hotmelt film at $100^{\circ} \mathrm{C}$ (Fig. $\left.1 \mathrm{~b}(\mathrm{iv})\right)$ an acetonitrile-based electrolyte that contains $\mathrm{I}^{-} / \mathrm{I}^{-}{ }_{3}$ redox couple (0.5 M KI, $0.05 \mathrm{M} \mathrm{I}_{2}$ in acetonitrile) is introduced through predrilled holes (Fig. 2a) and the device is sealed using the hotmelt film.

1. Bardeen, J. \& Brattain, W. The transistor, a semi-conductor triode. Phys. Rev. 74, 230-231 (1948).

2. Becker, J. A. \& Shive, J. N. The transistor - a new semiconductor amplifier. Electrical Engineering 68, 215-221 (1949).

3. Tanenbaum, M. et al. Silicon n-p-n grown junction transistors. J. Appl. Phys. 26, 686 (1955).

4. Mimura, T., Hiyamizu, S., Fujii, T. \& Nanbu, K. A new field-effect transistor with selectively doped GaAs/n- $\mathrm{Al}_{\mathrm{x}} \mathrm{Ga}_{1-\mathrm{x}} \mathrm{As}$ heterojunctions. Jpn. J. Appl. Phys. 19, L225-L227 (1980).

5. Mimura, T. Development of high electron mobility transistor. Jpn. J. Appl. Phys. 44, 8263-8268 (2005).

6. McCulloch, I. et al. Liquid-crystalline semiconducting polymers with high chargecarrier mobility. Nature Materials 5, 328-333 (2006).

7. Crone, B. et al. Large-scale complementary integrated circuits based on organic transistors. Nature 403, 521-523 (2000).

8. Rogers, J. A., Bao, Z. \& Raju, V. R. Nonphotolithographic fabrication of organic transistors with micron feature sizes. Appl. Phys. Lett. 72, 2716 (1998).

9. Li, S. et al. Polymer thin film transistors with self-aligned gates fabricated using ink-jet printing. Appl. Phys. Lett. 90, 172103 (2007).

10. Sekitani, T. et al. Organic nonvolatile memory transistors for flexible sensor arrays. Science 326, 1516-1519 (2009).
11. Yan, H. et al. A high-mobility electron-transporting polymer for printed transistors. Nature 457, 679-686 (2009).

12. Martel, R. et al. Single- and multi-wall carbon nanotube field-effect transistors. Appl. Phys. Lett. 73, 2447 (1998).

13. Tans, S. J., Verschueren, R. M. \& Dekker, C. Room-temperature transistor based on a single carbon nanotube. Nature 393, 49-52 (1998).

14. Lin, Y. M. et al. 100-GHz Transistors from wafer-Scale epitaxial graphene. Science 327, 662-662 (2010).

15. Britnell, L. et al. Field-effect tunneling transistor based on vertical graphene heterostructures. Science 335, 947-950 (2012).

16. Shockley, W. A unipolar field-effect transistor. Proc. IRE 40, 1365 (1952).

17. Tucker, J. R., Wang, C. \& Carney, P. S. Silicon field-effect transistor based on quantum tunnelling. Appl. Phys. Lett. 65, 618-620 (1994).

18. Nilsson, D. et al. Bi-stable and dynamic current modulation in electrochemical organic transistors. Adv. Mater. 14, 51 (2002).

19. Li, S., Chen, W., Chu, D. \& Roy, R. One step self-aligned multilayer patterning process for the fabrication of organic complementary circuits in combination with inkjet printing. Organic Electron. 13, 737-743 (2012).

20. Li, S., Chen, W., Chu, D. \& Roy, R. Self-aligned high-resolution printed polymer transistors. Adv. Mater. 23, 4107-4020 (2011)

21. Cantatore, E. et al. A 13.56-MHz RFID system based on organic transponders. IEEE Journal of solid-state circuits. 42, 84-92 (2007).

22. Zaumseil, J., Friend, R. H. \& Sirringhaus, H. Spatial control of the recombination zone in an ambipolar light-emitting organic transistor. Nature Materials 5, 69-74 (2006).

23. McCarthy, M. A. et al. Low-voltage, low-power, organic light-emitting transistors for active matrix displays. Science 332, 570-573 (2011).

24. Koneshan, S., Rasaiah, J. C., Lynden-Bell, R. M. \& Lee, S. H. Solvent structure, dynamics, and ion mobility in aqueous solutions at $25^{\circ} \mathrm{C}$. J. Phys. Chem. B 102, 4193-4204 (1998).

25. Bard, A. J. \& Faulkner, L. R. Electrochemical Methods: Fundamentals and Applications. (Wiley, New York, 2001).

26. Wang, X. Q. et al. Transistorlike behavior in photoconductor based on dyesensitized solar cell. Appl. Phys. Lett. 95, 011112 (2009).

27. Xu, J. et al. Steady-state transport characteristics of photoconductor based on dyesensitized solar cell. J. Appl. Phys. 107, 114511 (2010).

28. Wang, X., Xu, J., Liu, Z., Lu, Y. \& Cai, C. B. Low-voltage driving phototransistor based on dye-sensitized nanocrystalline titanium dioxide thin films. Curr. Appl. Phys. 12, 147-151 (2012).

29. Pattanasattayavong, P. et al. Solution-processed dye-sensitized $\mathrm{ZnO}$ phototransistors with extremely high photoresponsivity. J. Appl. Phys. 112, 074507 (2012).

30. Boschloo, G. et al. Research and development of dye-sensitized solar cells in the Center for Molecular Devices: from molecules to modules. World renewable energy congress, Sweden 2800-2806 (2011).

31. Mai, L., Dong, Y., Xu, L. \& Han, C. Single nanowire electrochemical devices. Nano Lett. 10, 4273-4278 (2010).

32. Yang, Y. et al. Flexible hybrid energy cell for simultaneously harvesting thermal, mechanical, and solar energies. ACS Nano 7, 785-790 (2013).

33. Qin, Y., Wang, X. \& Wang, Z. L. Microfibre-nanowire hybrid structure for energy scavenging. Nature 451, 809-813 (2008)

\section{Acknowledgments}

We thank financial support from Enterprise Ireland commercialization programme (Grant no: CF-2011-1606) and EU FP7 FET Flagship pilot program- 'Guardian angel for smarter life'. S.L. thanks Marie-Curie Fellowship (IEF) support.

\section{Author contributions}

S.L. conceived and designed the research. S.L., W.W. and J.X. have conducted device fabrication and test. Z.J.S., D.C., S.R. have contributed to experiment design and result analysis. S.L. and S.R. involved funding application and wrote the paper with the help of all others.

\section{Additional information}

Supplementary information accompanies this paper at http://www.nature.com/ scientificreports

Competing financial interests: The authors declare no competing financial interests.

How to cite this article: Li, S.P. et al. Electrical/optical dual-function redox potential transistor. Sci. Rep. 3, 3391; DOI:10.1038/srep03391 (2013).

cc) (i) $\odot$ This work is licensed under a Creative Commons Attributioncc) ${ }_{\mathrm{BY}} \mathrm{NC}$ ND NonCommercial-NoDerivs 3.0 Unported license. To view a copy of this license, visit http://creativecommons.org/licenses/by-nc-nd/3.0 